\title{
Targeted Vaccination Based on a Wireless Sensor System
}

\author{
Xiao Sun*, Zongqing Lu*, Xiaomei Zhang*, Marcel Salathé ${ }^{\dagger}$ and Guohong Cao* \\ ${ }^{*}$ Department of Computer Science and Engineering, The Pennsylvania State University, University Park, PA \\ ${ }^{\dagger}$ Department of Biology, The Pennsylvania State University, University Park, PA \\ $*\{$ xxs118, zongqing, xqz5057, gcao $\} @$ cse.psu.edu, ${ }^{\dagger}$ salathe@ @ psu.edu
}

\begin{abstract}
Vaccination is one of the most effective ways to protect people from being infected by infectious disease. However, it is often impractical to vaccinate all people in a community due to various resource constraints. Therefore, targeted vaccination, which vaccinates a small group of people, is an alternative approach to contain infectious disease spread. To achieve better performance in targeted vaccination, we collect student contact traces in a high school based on wireless sensors carried by students. With our wireless sensor system, we can record student contacts within the disease propagation distance, and then construct a disease propagation graph to model the infectious disease propagation. Based on this graph, we propose a metric called connectivity centrality to measure a node's importance during disease propagation and design centrality based algorithms for targeted vaccination. The proposed algorithms are evaluated and compared with other schemes based on our collected traces. Trace driven simulation results show that our algorithms can help to effectively contain infectious disease.
\end{abstract}

\section{INTRODUCTION}

One of the most effective ways to prevent infectious disease is to vaccinate the susceptible individuals. However, vaccinating all susceptible individuals is often impractical in many cases due to resource constraints such as the limited vaccine supply, especially during a new disease outbreak. Therefore, targeted vaccination, which vaccinates a small group of people in a community, is an alternative approach to contain infectious diseases. The challenge is to identify the subset of people whose vaccination will result, on average, in the maximal reduction of disease spread, given the resource constraints.

There has been some research on targeted vaccination [1] [2] [3]. However, these works are limited to theoretical study based on synthetic networks such as random, homogeneous or scale-free network, which may not reflect the real contact patterns among people.

The problem of targeted vaccination has some similarity to virus (worm) containment in the area of computer networks, such as cellular networks [4] [5] and online social networks [6] [7]. Based on cluster partition and community detection, several schemes have been proposed to select patching nodes for virus (worm) containment [5] [6]. The intuition behind these schemes is to divide the network into different partitions, and then contain the worms within the infected partition before they spread out. More specifically, the nodes that separate

This work was supported in part by the National Science Foundation (NSF) under grant number CNS-1421578. network partitions are vaccinated in [5] and the neighbors of overlapped nodes between two communities are vaccinated in [6]. However, these schemes implicitly assume that all nodes are eligible for vaccination, which is not true in disease containment. Additionally, the worm propagation model in cellular network or online social network is different from that of infectious disease, and thus these schemes cannot be directly applied to targeted vaccination.

Different from the aforementioned works, we collect student contact traces in a high school based on wireless sensors carried by students. Since the wireless signal strength degrades as the communication distance increases, we can measure the wireless signal strength and then infer when and where students meet with each other. This information is important for modeling the propagation of infectious disease. Many respiratory infectious diseases (e.g. influenza) spread from person to person by droplet transmission, requiring an infected and a susceptible person to be in close physical contact at a short maximum distance [8]. With our wireless sensor system, we can find student contacts within such distance, and construct a disease propagation graph to model the infectious disease propagation. Then, targeted vaccination becomes a problem of choosing important nodes in a graph to contain disease propagation.

Based on the disease propagation graph, node centrality can be used to measure its importance during disease propagation. There are various centrality measures [9] such as degree centrality, betweenness centrality and closeness centrality. Although these centrality metrics can be used to measure the importance of each node in disease propagation, they all have some disadvantages when applied for disease containment. For example, degree centrality only considers the connection between a node and its neighbors, and thus is limited by its local effect. Betweenness centrality measures the global effect, but the node's influence on its neighbors is treated equally as the influence on those nodes far away, which is not appropriate for disease propagation where nodes closer will be infected with much higher possibility than those far away. Thus, we propose a new metric called connectivity centrality, which considers a node's influence on all other nodes but assigns more weight on those closer. Then, we design centrality based algorithms for targeted vaccination. The proposed algorithms are evaluated and compared with other schemes based on the collected data trace. Trace driven simulation results show that 
our algorithms can significantly reduce the infection rate.

The rest of this paper is organized as follows. Section II describes our trace collection. Section III illustrates how to build the disease propagation graph. Centrality based algorithms for targeted vaccination are presented in Section IV, and the performance of our algorithms is evaluated in Section V. Section VI reviews related work and Section VII concludes the paper.

\section{TRACE Collection}

Most infectious diseases spread among people through virus, which is transmitted by airborne infectious particles or small respiratory droplets when two people contact within a certain distance [8]. However, most existing traces do not consider this limitation, and thus we collect our own traces in a high school.

\section{A. System Overview}

We use Crossbow TelosB motes to collect human contacts in a high school which has about 800 students. TelosB mote has USB programming capability, an IEEE 802.15.4 radio, and a low-power microcontroller with extended memory. Since the wireless signal strength degrades as the communication distance increases, we can measure the wireless signal strength and then infer when and where students meet with each other.

There are two types of motes in our system: mobile motes which are carried by students, and stationary motes which are deployed in some fixed locations for localization purpose. The mobile motes are placed in pouches, distributed to all the students at around 7 am and received back at around $4 \mathrm{pm}$ on each school day. Each mobile mote will be carried by a student around his (her) neck, and each mote has a unique ID. The mote broadcasts a beacon every 20 seconds and keeps listening to the wireless channel to record beacons from other motes. The beacon includes mote type, mote ID, and its local sequence number which is initialized to 0 and increased by one after each beacon broadcast. The stationary motes are deployed at certain locations such as classrooms, dining halls and restrooms. Each stationary mote broadcasts beacons with its mote type, ID and sequence number at an interval of 20 seconds with transmission power of $-11 \mathrm{dBm}$. The sequence number starts at 0 when the mote is powered on and increased by one after each broadcast. During trace collection, all the motes keep broadcasting beacons periodically and mobile motes record beacons from others. Beacons from mobile motes are recorded as contact information and beacons from stationary motes are recorded for time and location reference.

We deployed the sensor system and collected student contacts in two weeks during a flu season in March 2012. On average, there are about 3.4 million contacts between mobile motes on each day. Although student behaviors may vary across weekdays due to different curriculum schedules in different school days, on a weekly basis, the class schedules are repeated and student behaviors are similar.

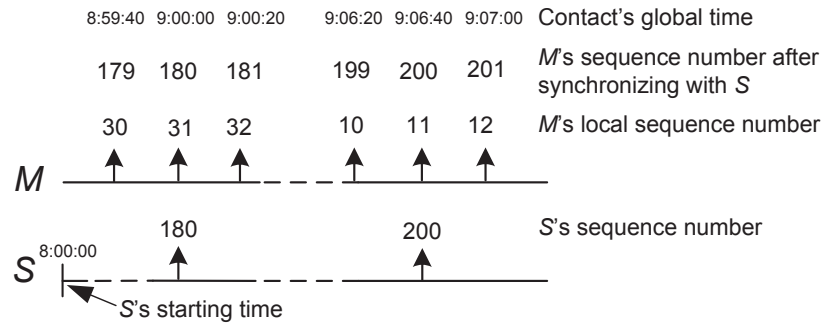

Fig. 1: The disordered sequence numbers received from mobile mote $M$ can be synchronized and converted to global time by exploiting the sequence numbers received from stationary mote $S$.

\section{B. Design Issues}

1) Disease Transmission Distance: The airborne droplets fail to transmit from one person to another if their contact distance is larger than 9 feet [8] [10]. From an epidemiological standpoint, 9 feet is a critical distance for disease propagation and thus we only need to collect contacts within this distance. In a wireless system, the received signal strength (RSSI) decreases as the distance between the sender and receiver increases. Therefore, we can determine if a contact happens within a specific range by checking if the corresponding RSSI is above certain threshold for a given transmission power.

In many sensor network applications, nodes are supposed to communicate with each other at the highest transmission power level to reach larger coverage area and achieve higher data delivery rate [11] [12]. However, our system is different, where only contacts within 9 feet are useful and thus it is not necessary to set mote with the highest transmission power. Also, a TelosB mote only has two AA batteries as its power supply. If it keeps working at the highest power level, the battery will quickly run out. Therefore, we choose a lower transmission power which does not affect the data reception rate. Our preliminary experimental results show that the transmission power of $-16.9 \mathrm{dBm}$ (power level 6 for TelosB mote) is strong enough to ensure a high data delivery rate within a distance of 9 feet. Under such transmission power and distance, the RSSI of the received packet is around -80 $\mathrm{dBm}$. Therefore, we set the transmission power of the mobile mote as $-16.9 \mathrm{dBm}$ and a beacon from the mobile mote is recorded only if its RSSI is larger than $-80 \mathrm{dBm}$. With such transmission power and RSSI threshold, we can ensure that the contacts collected in our traces are within 9 feet.

2) Global Time: The local sequence number of the beacon sent by a mobile mote is increased by one every 20 seconds. If its starting time $t_{0}$ is recorded, the global time $t_{g}$ of a contact with sequence number $l$ can be calculated as follows:

$$
t_{g}=t_{0}+20 s \times l
$$

However, as observed in the collected trace, most of the mobile motes' local sequence numbers do not increase monotonically due to node reboots caused by various factors, such as accidentally pressing reset button or loosening batteries. For example, as shown in Figure 1, after sequentially receiving beacons with sequence number 30,31 and 32 from mobile mote $M$, the mote receives another beacon with sequence 
number 10 from $M$. Apparently, $M$ suffered reboot and reinitialized its local sequence number to 0 during this period.

Since it is difficult to know when the reboot happened, the global time of the contact cannot be calculated merely by using the local sequence number of the mote. In our system, stationary motes are deployed in some fixed locations and broadcast periodically. They will not suffer from node reboots and will provide reliable sequence numbers, which can be used to synchronize the local sequence numbers of the mobile nodes and then to calculate the global time of the corresponding contact. As shown in Figure 1, if a mote receives beacon number 180 and 200 from the stationary mote $S$ when it gets beacon 31 and 11 from $M, M$ 's sequence number can be synchronized with $S$. Then, the global time of the contact can be calculated by using $S$ 's starting time.

In order not to disturb students' activities, the stationary motes are deployed at night before the trace collection and their starting times are recorded manually. In our system, each mobile mote receives about 4,500 beacons from stationary motes each day, which is enough for synchronizing the sequence number and calculating the global time. In this way, the global time for each contact can be obtained.

3) Indoor or Outdoor: According to [13], the infecting capability of infectious disease transmitted by droplets (e.g., influenza) varies in indoor and outdoor environment due to different air circulation patterns. Thus, it is important to know if the contact happens indoor or outdoor. The stationary motes are not only used for contact synchronization, but also used for indicating whether a contact happens indoor or outdoor. In our system, stationary motes are carefully deployed to cover the entire buildings in the school and thus if a mote is indoor at some time, it will receive beacons from at least one stationary mote at that time. Further, if a beacon is received from a mobile mote and at the same time both the sender and receiver have recorded beacons from some stationary motes, we can infer that this contact happens indoor; otherwise, it happens outdoor. Therefore, after synchronizing all traces, we can discern whether a contact happens indoor or outdoor by checking beacons received from the stationary motes.

\section{Disease Propagation Graph}

The collected contacts can be used to construct the disease propagation graph, which is used for modeling disease propagation. As shown in Figure 2, the disease propagation graph is an undirected weighted graph represented by $G=(V, E)$, where $V$ is the set of vertices and $E$ is the set of edges. Since disease infection is bidirectional, $G$ is an undirected graph. A node $u \in V$ represents a participant and an edge $(u, v) \in E$ exists only when there is contact between $u$ and $v$.

For each edge $(u, v)$, there is an associated weight $w(u, v)$, which denotes the disease propagation probability between these two nodes, and it is related to the contact frequency between them. For example, for two nodes that contact with each other frequently (i.e., they spend a lot of time together), if one node catches some infectious disease, the other one is most likely to be infected. In addition, the probability of infection is

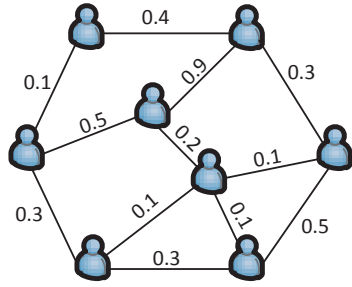

Fig. 2: Disease propagation graph

related to where the node contact happens. According to [13], infectious disease such as influenza, is more likely to spread quickly in indoor environment than outdoor environment.

Considering both contact frequency and contact location, the edge weight $w(u, v)$ is calculated as follows:

$$
w(u, v)=\frac{\sum_{t=0}^{T} \eta(u, v, t) r(u, v, t)}{T}
$$

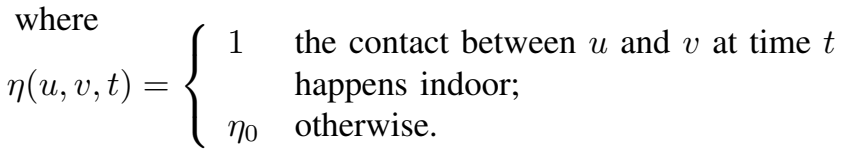

$r(u, v, t)= \begin{cases}1 & \text { if there is a contact between } u \text { and } v \text { at } t ; \\ 0 & \text { otherwise. }\end{cases}$

and $T$ is a time period of the trace.

Since infectious disease is relatively inactive in outdoor environment, $\eta(u, v, t)$ is set to $\eta_{0}\left(0<\eta_{0}<1\right)$ if the contact happens outdoor and the value of $\eta_{0}$ depends on the characteristic of the specific disease.

Whenever there is a contact between two nodes, there is some probability for the infectious disease to be transmitted between them. This disease propagation graph shows how each node contacts with others and how disease propagates among them.

\section{Centrality Based Targeted Vaccination}

In targeted vaccination, a small set of nodes are chosen to get vaccine to mitigate disease propagation. With $k$ vaccine, one naive solution is to randomly pick $k$ nodes to be vaccinated. However, this is not a good solution because each node has different influence on others and thus plays a different role during disease propagation. The importance of each node on disease propagation can be measured by centrality. In this section, we first introduce some well known centrality metrics [9] and then propose a new metric called connectivity centrality, based on which we design centrality based algorithm for targeted vaccination.

\section{A. Node Centrality}

There are various centrality measures such as degree, betweenness, and closeness, which are defined as follows.

Degree centrality: Let $N(u)$ denote the set of $u$ 's neighboring nodes, then $u$ 's degree centrality is:

$$
C_{d}(u)=\sum_{v \in N(u)} w(u, v)
$$

Betweenness centrality: Let $\sigma_{s t}$ denote the total number of shortest paths from node $s$ to $t$; let $\sigma_{s t}(u)$ denote the total 
number of shortest paths from node $s$ to $t$ that go through node $u$, then $u$ 's betweenness centrality is:

$$
C_{b}(u)=\sum_{s \neq u \neq t, s, t \in V} \frac{\sigma_{s t}(u)}{\sigma_{s t}}
$$

Closeness centrality: Let $d(u, v)$ denote the shortest path distance between $u$ and $v$, then node $u$ 's closeness centrality is:

$$
C_{c}(u)=\frac{|V|-1}{\sum_{v \neq u, v \in V} d(u, v)}
$$

Generally speaking, degree centrality measures how well a node is connected with its neighbors; betweenness centrality measures to what extent a node can connect two other nodes through a shortest path; closeness centrality measures how close a node is to others. Although these centrality metrics can be used to measure the importance of each node in disease propagation, they all have some disadvantages when applied for disease containment. Degree centrality only considers the connection between a node and its neighbors, and thus is limited by its local effect. Betweenness centrality measures the global effect, but the node's influence on its neighbors is treated equally as the influence on those nodes far away, which is not appropriate for disease propagation where nodes closer will be infected with much higher possibility than those far away. By considering distance, closeness centrality treats nodes differently based on their distance. However, since the distance is simply added together, the path with longer distance may dominate the result. Further, if nodes $u$ and $v$ are in different partitions of a disconnected graph, $d(u, v)=\infty$. According to its definition, $C_{c}(u)$ will be 0 no matter how close it is to other nodes. Thus, closeness centrality can only be used in a connected graph.

\section{B. Connectivity Centrality}

To better measure how contagious an infected node is to others, we propose a new centrality metric called connectivity centrality, which is defined as follows:

$$
C_{\text {con }}(u)=\sum_{v \neq u, v \in V} c(u, v)
$$

where

$$
c(u, v)= \begin{cases}\frac{1}{d(u, v) h(u, v)} & \text { if there is a path from } u \text { to } v ; \\ 0 & \text { otherwise. }\end{cases}
$$

and $h(u, v)$ denotes the number of hops between $u$ and $v$ along the shortest path.

Connectivity centrality considers a node's effect on others and assigns more weight on nodes closer and within less hops. This is because infectious disease is more likely to be transmitted to nearby nodes within limited hops. Figure 3 illustrates the difference between degree centrality, betweenness centrality and connectivity centrality. Since closeness centrality can only be used in a connected graph, we do not consider it here. Except degree centrality, all other centralities are distance based and the weight between any two neighboring nodes represents their distance. However, in the disease propagation

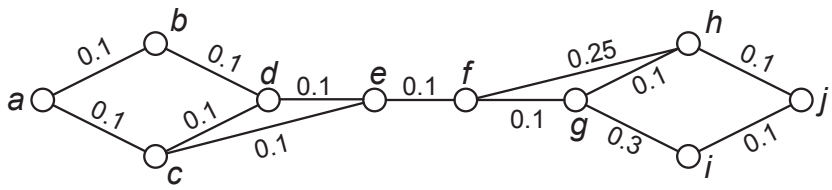

Fig. 3: An example of different centrality metrics in the disease propagation graph. Node $g$ has the highest degree centrality 0.5 ; both node $e$ and node $f$ have the highest betweenness centrality 40; node $f$ has the highest connectivity centrality 0.595 .

graph, the edge weight is assigned based on the disease propagation probability. The larger the edge weight is, the closer the nodes are and the smaller their distance is. Therefore, when calculating distance based centralities, $\frac{1}{w(u, v)}$ is used as the distance between two neighboring nodes $u$ and $v$.

As shown in Figure 3, different node centralities (degree, betweenness, connectivity) are used to remove one node to contain the disease. Node $g$ has the highest degree centrality. However, it is not the best choice for vaccination since the residual graph is still connected after its removal. Both node $e$ and node $f$ have the highest betweenness centrality, and removing either of them will separate the graph into two parts, which can stop the disease spreading from one partition to another. Comparing $e$ and $f$, node $f$ which has the highest connectivity centrality is better, because its removal will also remove edge $(f, h)$ which has a larger edge weight and is easier to transmit disease. In this example, node $g$ only has influence on its neighbors; node $e$ is globally important; node $f$ is both locally and globally important. Therefore, connectivity centrality is better than other centrality metrics for measuring node's influence on disease propagation.

\section{Centrality Based Algorithm}

Node centrality describes how a node connects with others. Thus, nodes with higher centrality will be more likely to infect others once they are infected and these nodes should be given higher priority for vaccination. To find these nodes, one simple solution is to sort them based on their centrality and select the highest $k$ nodes to be vaccinated. However, in disease containment, not all nodes are eligible for vaccination. For example, if a node has already been infected, vaccinating it will not be effective. Also, some nodes may refuse to get vaccinated due to concerns on the effectiveness of the vaccine or potential side effects [14]. Therefore, after sorting, the first $k$ nodes which are eligible to get vaccinated are picked as the targeted nodes.

\section{Adaptive Algorithm}

Although centrality based algorithm is simple, it may not achieve the best performance since each node's centrality is calculated based on the original graph. However, once a node is selected to be vaccinated, it will be removed from the graph and change the graph topology. Thus, the centrality value for the remaining nodes should be calculated based on the updated graph. To address this problem, we propose an adaptive centrality based algorithm. At each round, all nodes are sorted based on their updated centrality values and the one 
with the highest centrality is chosen as the candidate node. If this node is qualified for vaccination, it is removed from the graph, and the node's centrality is recalculated based on the updated graph; otherwise, the node with the next highest centrality is chosen. This process is repeated until $k$ nodes are selected for vaccination. The pseudo code of the algorithm is shown in Algorithm 1.

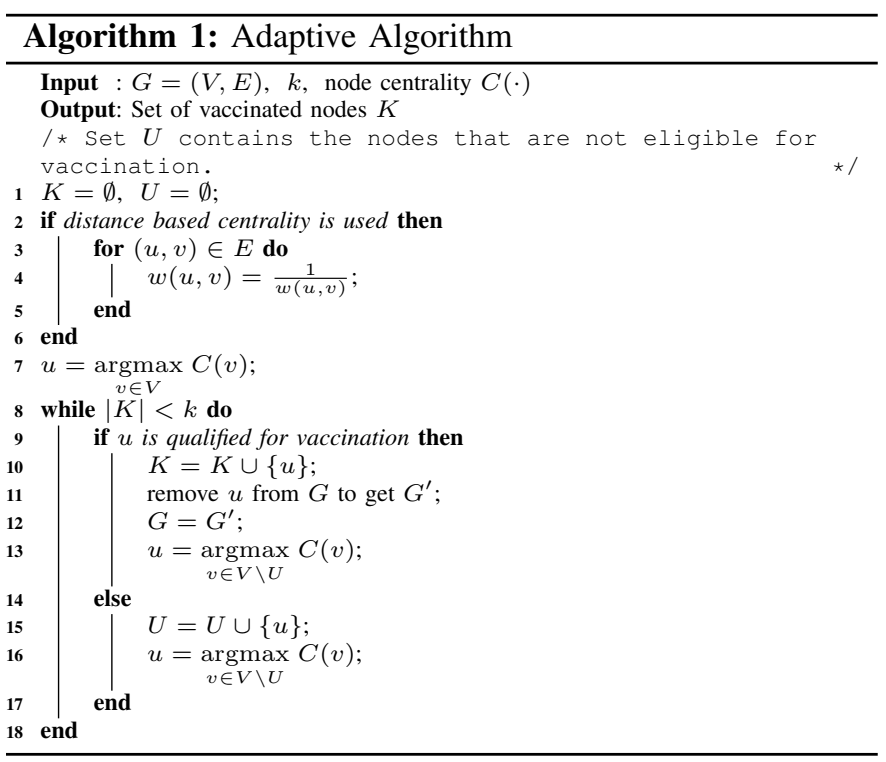

\section{PERformance Evaluations}

In this section, we evaluate the performance of our algorithms and compare them to other schemes.

\section{A. Simulation Setup}

The evaluations are based on our trace collected in a high school. The first half of the trace is used as the training data to build the disease propagation graph, and the second half is used for performance evaluations.

At the beginning, a small group of nodes (1\%) are randomly chosen as the seed set of infection sources. The trace is executed based on time units. At each time unit, the SIR model [15] is used to simulate the infection process. In SIR, each node has three states: $\mathbf{S}$ (Susceptible), I (Infected) and $\mathbf{R}$ (Recovered). Suppose a node is initially at $\mathbf{S}$ state. By contacting with an infected node, it will be infected with probability $\beta$ (called transmission probability) indoor and $\eta_{0} \beta$ ( $\eta_{0}$ is fixed at 0.5 in our simulations) outdoor. If the node is infected, it will move into state $\mathbf{I}$. An infected node may recover with a probability $\delta$ ( $\delta$ is fixed at 0.0003 in our simulations) at each time unit and goes back to state $\mathbf{R}$. Nodes who are in state $\mathbf{R}$ have already got immunization and will not be infected again.

Our centrality based algorithms and adaptive centrality based algorithm are compared with the overlapping community detection based scheme (AFOCS) [6] and the cluster based scheme (Cluster) [5]. Degree centrality, betweenness centrality and connectivity centrality are used to implement centrality based algorithm (denoted as Degree, Betweenness

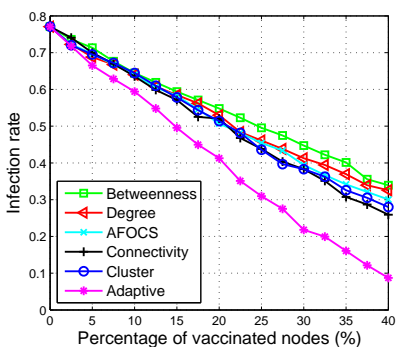

(a) $\alpha=2.5 \%$

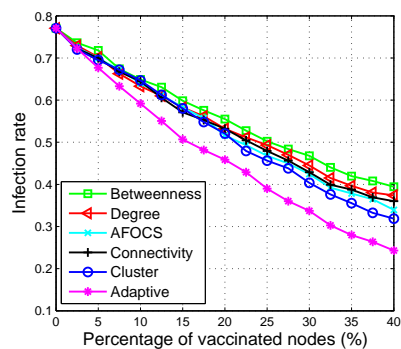

(b) $\alpha=10 \%$
Fig. 4: Effect of vaccinating threshold $\alpha(\beta=0.003)$

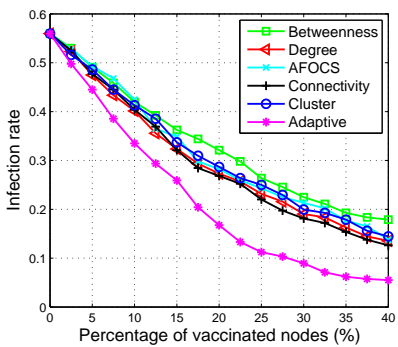

(a) $\beta=0.002$

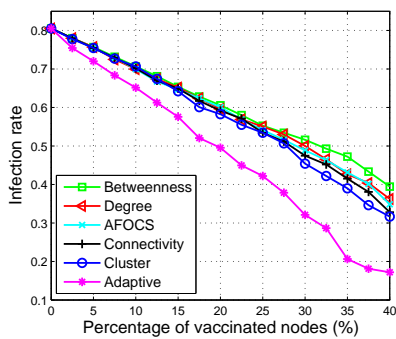

(b) $\beta=0.004$
Fig. 5: Effect of the disease transmission probability $\beta(\alpha=2.5 \%)$

and Connectivity respectively). Connectivity centrality is used to implement the adaptive algorithm (denoted as Adaptive). Closeness centrality is not used here since it does not work when the graph is disconnected.

We use a Vaccinating Threshold $\alpha$ to control when the targeted vaccination starts. It is measured as the percentage of infected nodes in the network. This parameter represents the time delay since the infectious disease starts propagating till it is detected and a vaccine is generated. Once the percentage of infected nodes reaches this threshold $\alpha$, we start to distribute vaccines to the chosen nodes.

\section{B. Comparisons of Infection Rates}

Figure 4 shows how the infection rate changes when the percentage of vaccinated nodes increases with $\alpha=2.5 \%$ and $10 \%$ respectively. As can be seen, for all schemes, fewer nodes will be infected when more vaccines are distributed. Adaptive outperforms other schemes under different $\alpha$. When $\alpha=2.5 \%$, with $20 \%$ of nodes vaccinated, the infection rate of Adaptive is about $40 \%$, but the infection rates of other schemes are higher than $50 \%$. For the centrality based algorithms, under different $\alpha$, Degree performs better than Betweenness since disease is easier to transmit from the infected nodes to their neighbors than to those far away. Connectivity performs better than Betweenness and Degree, verifying that connectivity centrality is better to measure node's importance for disease propagation.

Comparing Figure $4 \mathrm{a}$ with Figure $4 \mathrm{~b}$, we can see that AFOCS and Cluster perform worse than Connectivity when $\alpha=2.5 \%$, but better when $\alpha=10 \%$. The reason is as follows. If more nodes are infected before vaccination (i.e., $\alpha$ is larger), these infected nodes are more likely to be clustered together around the infection nodes. Since AFOCS and Cluster contain the disease by isolating infected communities or clusters, they 


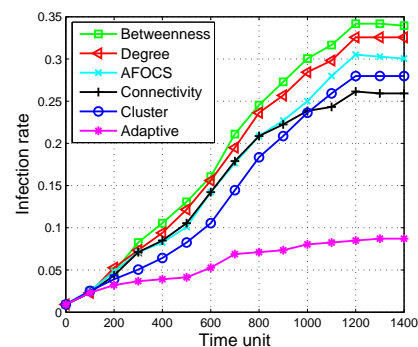

(a) $\alpha=2.5 \%$

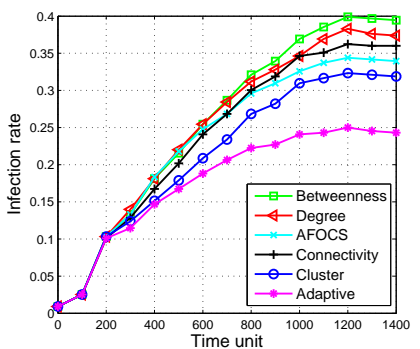

(b) $\alpha=10 \%$
Fig. 6: Infection rate vs. time (percentage of vaccinated nodes $=40 \%$, $\beta=0.003$ )

can perform better. However, their infection rate is still much higher than the proposed Adaptive.

\section{Effect of Transmission Probability}

Figure 5 shows how the disease transmission probability $\beta$ affects the spread of disease. As can be seen, Adaptive performs better than other schemes. For centrality based algorithms, Connectivity outperforms Degree and Betweenness under various $\beta$. Comparing centrality based algorithms with Cluster, both Connectivity and Degree perform better than Cluster when $\beta=0.002$, but Cluster performs better than Connectivity and Degree when $\beta=0.004$. The reason is as follows. Generally speaking, if the infected nodes are uniformly distributed, centrality based algorithms will perform better; if the infected nodes are clustered together, Cluster performs better. With a lower $\beta$, nodes will be infected more randomly, and then their distribution looks more uniform. With a higher $\beta$, nodes with close connections will be infected more easily and thus the infected nodes are more likely to be clustered together.

\section{Infection Rate vs. Time}

Figure 6 shows how the infection rate changes over time with $\alpha=2.5 \%$ and $10 \%$, respectively. The spread of the disease can be divided into three phases. At the beginning, the disease is slowly spread from the infection sources. Then, it propagates widely and the infection rate increases quickly. Finally, no more nodes will get infected and the infection rate keeps stable. Adaptive achieves better performance than other schemes as their infection rate is increased more slowly and the infection rate is bounded under a much lower level.

\section{RELATED WORK}

There have been lots of research on infectious disease containment. In [1], Madar et al. studied vaccination strategies under the SIR model for scale-free graphs. Hayashi et al. [2] studied the case of growing networks and derived conditions for extinction under random vaccination and targeted vaccination. However, these works are based on synthetic networks.

By extracting the communication patterns of mobile devices based on a cellular network trace, Zhu et al. [5] proposed cluster based algorithms for mitigating MMS (Multimedia Messaging Service) worms. In [6], Nguyen et al. proposed AFOCS to detect overlapping communities in a network and selected the nodes in the boundary of the overlapped regions as the patching targets to prevent worms from spreading out in online social network. However, the worm propagation model in cellular network or online social network is different from that of infectious disease, and thus these schemes cannot be directly applied to targeted vaccination.

\section{CONCLUSIONS}

In this paper, we designed and deployed a system to collect student contact traces in a high school based on wireless sensors carried by students. With our wireless sensor system, we can find student contacts within the disease propagation distance, and construct a disease propagation graph to model the infectious disease propagation. Then, we proposed the metric of connectivity centrality to measure a node's importance during disease propagation and designed centrality based algorithms for targeted vaccination. Through trace driven evaluations, we demonstrated that our algorithms can help to effectively contain infectious disease and significantly outperform other existing schemes.

\section{REFERENCES}

[1] N. Madar, T. Kalisky, R. Cohen, D. Ben-avraham, and S. Havlin, "Immunization and epidemic dynamics in complex networks," The European Physical Journal B-Condensed Matter and Complex Systems, vol. 38, no. 2, pp. 269-276, 2004.

[2] Y. Hayashi, M. Minoura, and J. Matsukubo, "Recoverable prevalence in growing scale-free networks and the effective immunization," arXiv preprint cond-mat/0305549, 2003.

[3] Y. Wang, G. Xiao, J. Hu, T. H. Cheng, and L. Wang, "Imperfect targeted immunization in scale-free networks," Physica A: Statistical Mechanics and its Applications, vol. 388, no. 12, pp. 2535-2546, 2009.

[4] F. Li, Y. Yang, and J. Wu, "Cpmc: An efficient proximity malware coping scheme in smartphone-based mobile networks," in IEEE INFOCOM, 2010.

[5] Z. Zhu, G. Cao, S. Zhu, S. Ranjan, and A. Nucci, "A social network based patching scheme for worm containment in cellular networks," in IEEE INFOCOM, 2009.

[6] N. Nguyen, T. Dinh, S. Tokala, and M. Thai, "Overlapping communities in dynamic networks: their detection and mobile applications," in $A C M$ Mobicom, 2011

[7] Z. Lu, Y. Wen, and G. Cao, "Community detection in weighted networks: Algorithms and applications," in IEEE Percom, 2013.

[8] V. C. Barclay, T. Smieszek, J. He, G. Cao, J. J. Rainey, H. Gao, A. Uzicanin, and M. Salathé, "Positive network assortativity of influenza vaccination at a high school: implications for outbreak risk and herd immunity," PloS one, vol. 9, no. 2, p. e87042, 2014.

[9] L. C. Freeman, "Centrality in social networks conceptual clarification," Social networks, vol. 1, no. 3, pp. 215-239, 1979.

[10] M. Kazandjieva, J. Lee, M. Salathé, M. Feldman, J. Jones, and P. Levis, "Experiences in measuring a human contact network for epidemiology research," in Proceedings of the 6th Workshop on Hot Topics in Embedded Networked Sensors. ACM, 2010.

[11] J. E. Bardram, A. Doryab, R. M. Jensen, P. M. Lange, K. L. Nielsen, and S. T. Petersen, "Phase recognition during surgical procedures using embedded and body-worn sensors," in IEEE PerCom, 2011.

[12] R. Zhou, G. Xing, X. Xu, J. Wang, and L. Gu, "Wiznet: A zigbee-based sensor system for distributed wireless lan performance monitoring," in IEEE PerCom, 2013.

[13] T. Weber and N. Stilianakis, "Inactivation of influenza a viruses in the environment and modes of transmission: a critical review," Journal of Infection, vol. 57, no. 5, pp. 361-373, 2008.

[14] M. Pareek, T. Clark, H. Dillon, R. Kumar, and I. Stephenson, "Willingness of healthcare workers to accept voluntary stockpiled h5n1 vaccine in advance of pandemic activity," Vaccine, vol. 27, 2009.

[15] H. Hethcote, "The mathematics of infectious diseases," SIAM review, vol. 42, no. 4, pp. 599-653, 2000. 